\title{
Automated Hypofractionated IMRT treatment planning for early-stage breast Cancer
}

Ting-Chun Lin ${ }^{1 \dagger}$, Chih-Yuan Lin ${ }^{1 \dagger}$, Kai-Chiun Li ${ }^{1}$, Jin-Huei Ji ${ }^{1}$, Ji-An Liang ${ }^{1,2}$, An-Cheng Shiau ${ }^{1,3,4}$, Liang-Chih Liu ${ }^{2,5}$ and Ti-Hao Wang ${ }^{1 *}$

\begin{abstract}
Background: Hypofractionated whole-breast irradiation is a standard adjuvant therapy for early-stage breast cancer. This study evaluates the plan quality and efficacy of an in-house-developed automated radiotherapy treatment planning algorithm for hypofractionated whole-breast radiotherapy.

Methods: A cohort of 99 node-negative left-sided breast cancer patients completed hypofractionated whole-breast irradiation with six-field IMRT for 42.56 Gy in 16 daily fractions from year 2016 to 2018 at a tertiary center were replanned with an in-house-developed algorithm. The automated plan-generating C\#-based program is developed in a Varian ESAPI research mode. The dose-volume histogram (DVH) and other dosimetric parameters of the automated and manual plans were directly compared.

Results: The average time for generating an autoplan was 5 to $6 \mathrm{~min}$, while the manual planning time ranged from 1 to $1.5 \mathrm{~h}$. There was only a small difference in both the gantry angles and the collimator angles between the autoplans and the manual plans (ranging from 2.2 to 5.3 degrees). Autoplans and manual plans performed similarly well in hotspot volume and PTV coverage, with the autoplans performing slightly better in the ipsilateral-lungsparing dose parameters but were inferior in contralateral-breast-sparing. The autoplan dosimetric quality did not vary with different breast sizes, but for manual plans, there was worse ipsilateral-lung-sparing $\left(\mathrm{V}_{4 \mathrm{~Gy}}\right)$ in larger or medium-sized breasts than in smaller breasts. Autoplans were generally superior than manual plans in $\mathrm{Cl}(1.24 \pm$ 0.06 vs. $1.30 \pm 0.09, p<0.01)$ and $M U(1010 \pm 46$ vs. $1205 \pm 187, p<0.01)$.

Conclusions: Our study presents a well-designed standardized fully automated planning algorithm for optimized whole-breast radiotherapy treatment plan generation. A large cohort of 99 patients were re-planned and retrospectively analyzed. The automated plans demonstrated similar or even better dosimetric quality and efficacy in comparison with the manual plans. Our result suggested that the autoplanning algorithm has great clinical applicability potential.
\end{abstract}

Keywords: Automation, Treatment planning, Autoplanning, Hypofractionation, IMRT, Early-stage, Whole-breast irradiation, Left-sided breast cancer

\footnotetext{
* Correspondence: thothwang@gmail.com

†Ting-Chun Lin and Chih-Yuan Lin contributed equally to this work.

'Department of Radiation Oncology, China Medical University Hospital, China

Medical University, Taichung, Taiwan

Full list of author information is available at the end of the article
}

(c) The Author(s). 2020 Open Access This article is distributed under the terms of the Creative Commons Attribution 4.0 International License (http://creativecommons.org/licenses/by/4.0/), which permits unrestricted use, distribution, and reproduction in any medium, provided you give appropriate credit to the original author(s) and the source, provide a link to the Creative Commons license, and indicate if changes were made. The Creative Commons Public Domain Dedication waiver (http://creativecommons.org/publicdomain/zero/1.0/) applies to the data made available in this article, unless otherwise stated. 


\section{Background}

Breast cancer is the second most prevalent cancer and the second leading cause of cancer death among women worldwide, with a slight but stable increase in its incidence in the most recent decade [1]. At our center, breast cancer patients account for approximately onethird of all patients requiring curative radiotherapy. Fortunately, most patients are diagnosed at early stage without nodal involvement, and for this subgroup, the standard treatment consists of surgery and postoperative radiotherapy, and systemic adjuvant therapy if necessary. Postoperative whole-breast irradiation serves as an adjuvant therapy following breast-conserving surgery that provides equivalent long-term survival comparable to radical mastectomy, and is now recognized as the standard treatment for early-stage breast cancer [2]. For node-negative patients, the hypofractionated schedule is commonly recommended as randomized trials have confirmed their safety and efficacy [3]. At our department, hypofractionated radiotherapy (42.56 Gy in 16 fractions) has been implemented for nearly all early-stage nodenegative breast cancer patients in the recent 2 years.

Following the Quantitative Analysis of Normal Tissue Effects in the Clinic (QUANTEC) and Radiation Therapy Oncology Group (RTOG) consensus guidelines, the main organs at risk (OARs) for whole-breast radiotherapy include the heart, lung and contralateral breast [4, 5]. Whole-breast irradiation is generally performed with two opposed tangential beams to avoid the abovementioned normal organs while simplifying the treatment plan. Tangential beam intensity-modulated radiation therapy (IMRT) may be employed to enhance the dose homogeneity [6-9]. Compared with other cancer sites, treatment planning for whole-breast irradiation is relatively simple yet contributes to a large proportion of the workload for the medical personnel in radiation oncology departments, and thus is an ideal candidate for automation. In the recent decade several published studies reported on automated treatment planning algorithms implementation for the head and neck, tangent breast, prostate and palliative spine [10-15]. These automated planning algorithms reduced the time and effort required to create personalized treatment plans, while also allowing the planning process to be highly standardized. The published studies generally showed similar target coverage and equivalent clinical acceptability of automated plans when compared with manual plans. For head and neck cancer planning, better OAR sparing favoring the automated plans were even reported [12].

We argue that a well-designed automated planning algorithm can improve current radiation oncology clinical workflow by standardizing plan quality, accelerating the treatment planning process, and improving employee productivity. We collected left-sided breast cancer patient manual treatment plans who received hypofractionated whole-breast irradiation as an adjuvant therapy for partial mastectomy at our hospital to prove this concept. These treatment plans were re-planned with an inhouse-developed algorithm. The dosimetric quality was compared between the automated plans and manual plans.

\section{Methods \\ Patient selection}

A cohort of 99 patients diagnosed with left-sided stage I or stage II node-negative breast cancer who received postoperative whole-breast irradiation without nodal irradiation from year 2016 to 2018 at a tertiary center were included in this study. All patients were aged 20 years or older and received hypofractionated regimen of $42.56 \mathrm{~Gy}$ in 16 daily fractions.

\section{CT simulation and contouring}

A customized immobilization device was used for all patients with the ipsilateral arm raised to maximize the precision and repeatability of the daily positioning for irradiation. Following patient immobilization, planning images were acquired with a computerized tomography (CT) scanner (SOMATOM Definition AS, Siemens, Germany) at a 3-mm slice thickness. Our department used the voluntary deep-inspiration breath-hold (DIBH) technique to reduce the cardiac radiation dose in breast cancer management. The patients performed a supervised breath hold during CT simulation and treatment.

For each patient in our study cohort, we re-planned with our auto-planning algorithm, based on previous approved contours. The treatment target and OARs were manually contoured based on the RTOG 1005 protocol guidelines [16]. The clinical target volume (CTV) included the whole left breast and was extended isotropically with a margin of $5 \mathrm{~mm}$ to form the planning target volume (PTV). The PTV was cropped to a distance of 4 $\mathrm{mm}$ from the patient's skin surface. The contralateral breast, ipsilateral and contralateral lung, and heart were manually contoured as OARs.

\section{Manual treatment planning technique}

All manual plans for hypofractionated whole-breast irradiation used six-field IMRT design with six MV photon beams at our department, created by experienced medical physicists. Two tangential beams (major fields) were manually assigned by the treatment planner to fit the PTV borders. Four oblique beams (minor fields) with reduced field size were subsequently created. All of the fields were extended at the breast apex to account for the breathing motion. The minor beam angles were adjusted to avoid the critical organs. Dose-volume constraints were set based on the RTOG 1005 protocol. 
Auxiliary structures Ring_1 and Ring_2 were manually created by the treatment planner. The areas of relative overdose (hotspots) were eliminated by assigning the hot spots region, isodose area of $\mathrm{V}_{105 \%}$, as a constraint structure to the objective template. The elimination of hotspots usually needs to be repeated several times. A beam's-eye-view example of the manual plan is shown in Fig. 1.

\section{Automatic treatment plan (autoplan) generation}

The automated plan-generating computer algorithm is an in-house-developed C\# program created as a script of the Varian Eclipse Scripting Application Programming Interface (ESAPI). The generated autoplan schematic diagram is shown in Fig. 2. The detailed process is described as follows: (1) Automatically create auxiliary structures Ring_1 and Ring_2 by extending the PTV posteriorly (Fig. 1), and then create a new plan under a new course for a selected patient. (2) Set the centroid of the PTV as the treatment isocenter. (3) Identify the optimal opposed tangential gantry angles and collimator angles by iterating the angles one degree at a time. For the gantry angle, iterate between 270 and 330 degrees (300 \pm 30 degrees range) for major field F1, and between 90 and 150 degrees (120 \pm 30 degrees range) for major field F2. For the collimator angle, iterate between 330 and 30 degrees $(0 \pm 30$ degrees range). For each iteration, the jaw fits to the PTV and then the field area (defined by the $\mathrm{X}$ and $\mathrm{Y}$ jaws) is calculated. We choose the gantry and collimator angles pair which has the smallest field area. (4) Add four minor fields F3, F4, F5 and F6. The three minor fields F3, F4 and F5 are set based on F1. The gantry angles are set as follows: $\mathrm{F} 3=\mathrm{F} 1+15$ degrees, $\mathrm{F} 4=\mathrm{F} 1+30$ degrees, $\mathrm{F} 5=\mathrm{F} 1+45$ degrees, and the collimator angles of these three subfields are set as F1. The fourth minor field F6 is determined based on F2, with F6 gantry angle $=$ F2 -15 degrees, and F6 collimator angle is set as F2. For the minor fields, the jaws fit to the PTV, and then the inner side of the jaw opens $1.5 \mathrm{~cm}$ away from the isocenter (Fig. 1). (5) Optimize plan according to the objective template (Additional file 1: Table S1) and calculate dose. (6) Evaluate and minimize $\mathrm{V}_{105 \%}$ hotspot area. If $\mathrm{V}_{105 \%}<0.5 \mathrm{cc}$, the plan is completed (Step 8). If $\mathrm{V}_{105 \%}>0.5 \mathrm{cc}$, the constraint structure
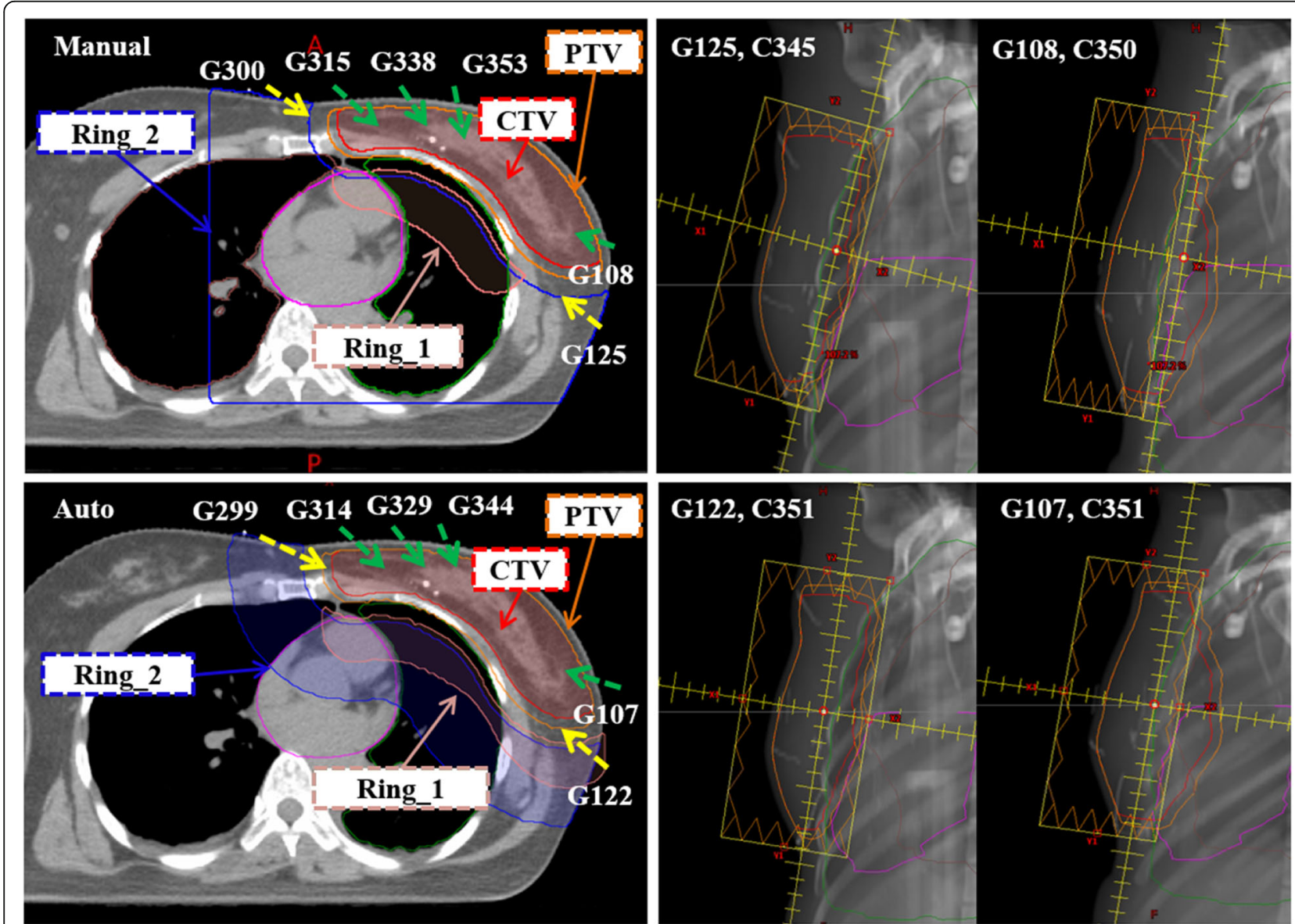

Fig. 1 A representative CT axial view demonstrating beam arrangement and planning auxiliary structure (left panel) and beam's-eye-view (right panel). Upper row: manual plan. Lower row: autoplan 


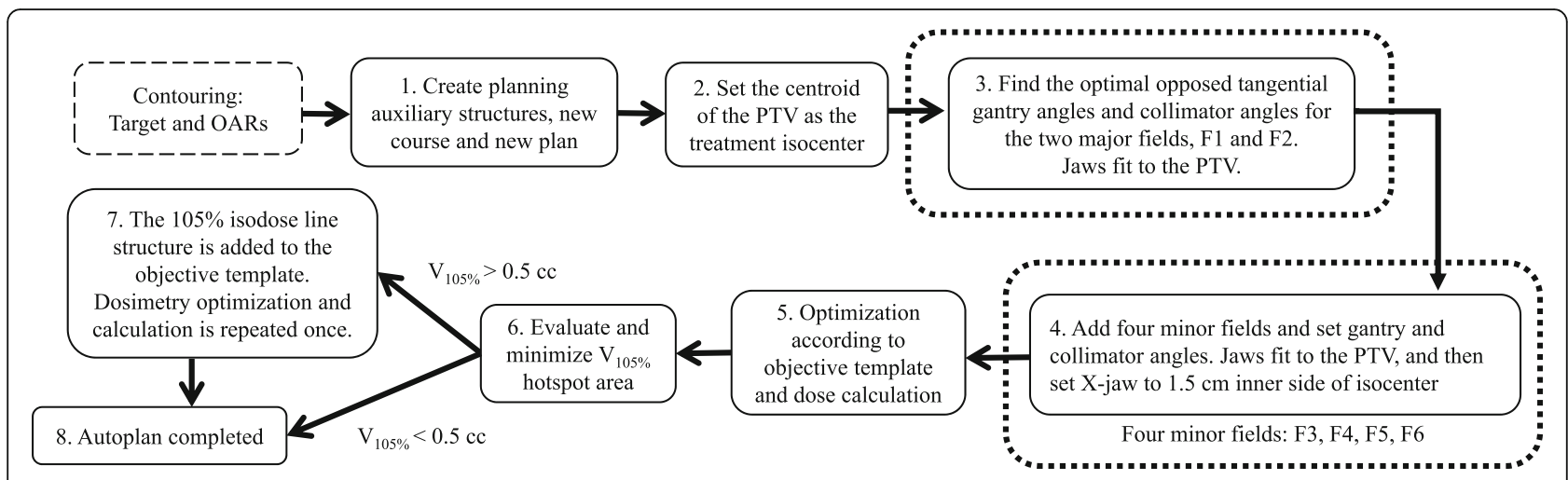

Fig. 2 The schematic diagram of an autoplan. Detailed description is in the Methods and Materials section

with region of $105 \%$ isodose area will be automatically created, and the program moves to the next step (Step 7). (7) The $V_{105 \%}$ constraint structure is added to the objective template (Additional file 1: Table S1). Plan optimization and dose calculation are repeated once again. (8) The autoplan is completed.

All automated plans were generated using the Eclipse treatment planning system (TPS) under a research license (Version 15.6, Varian Medical Systems Inc., Palo Alto, California, USA). Analytical Anisotropic Algorithm was used for dose calculation for all plans. All automated plans were simulated on the TPS but not delivered clinically.

\section{Plan evaluation and analysis}

The DVHs and dosimetric parameters of all manual plans and autoplans were collected and calculated for data analysis. The mean DVH band was calculated and reflected the volume-dose distribution with $95 \%$ confidence interval. For plan evaluation, the following parameters were recorded. The $V_{110 \%}$ of the prescribed dose inside the body was analyzed for hotspot evaluation. For PTV coverage evaluation, $V_{95 \%}$ was collected. The dose homogeneity index $(\mathrm{HI})$ of PTV was measured by $\mathrm{D}_{5 \%}$ divided by $\mathrm{D}_{95 \%}\left(\mathrm{D}_{5 \%} / \mathrm{D}_{95 \%}\right)$ [17]. The conformity index (CI) of PTV was defined as $\mathrm{BV}_{95 \%} / \mathrm{PTV}\left(\mathrm{BV}_{95 \%}=\right.$ the volume of the body receiving $95 \%$ of the prescribed dose) [18]. For dose analysis of the OARs, the $\mathrm{V}_{16 \mathrm{~Gy}}$ and $D_{\text {mean }}$ were reported for the ipsilateral lung, $V_{20 G y}$ and $D_{\text {mean }}$ for the heart, and $V_{5 G y}$ and $D_{\max }$ for the contralateral breast per RTOG 1005 protocol. The monitor unit (MU) was calculated by summing the MUs of all fields in a treatment plan.

\section{Statistical analysis}

The dosimetric parameters and total MUs were evaluated by paired two-tailed $t$-test for the manual and automatic treatment plans. One-way analysis of variance (ANOVA) was applied to evaluate the differences between OAR parameters of patients with different breast sizes stratified into three groups (small, medium and large) according to the CTV with a cutoff of $300 \mathrm{ml}$ and $600 \mathrm{ml}$. A $p$-value less than 0.05 was considered statistically significant. Data are presented as mean value \pm standard deviation (SD) if not otherwise specified. All statistical analyses and graphs were performed using the statistical software $\mathrm{R}$ version 3.5.2.

\section{Results}

The mean difference between the gantry and collimator angles for F1 and F2 fields between autoplans and manual plans were $2.69 \pm 3.00,5.24 \pm 5.63,2.26 \pm 2.67$ and $2.42 \pm 2.81$ (Mean $\pm \mathrm{SD}$ ) degrees, respectively. For hotspot $\mathrm{V}_{105 \%}$ evaluation of all 99 autoplans, 66 (67.7\%) plans had smaller $\mathrm{V}_{105 \%}$ after the automatic optimization step (Step 6), 24 (24.2\%) did not have $V_{105 \%}>0.5 \mathrm{cc}$, and eight (8.1\%) plans still had $V_{105 \%}>0.5 \mathrm{cc}$ after optimization.

Table 1 listed the dosimetric parameters for the hotspot area $\left(\mathrm{V}_{110 \%}\right)$, the PTV coverage, ipsilateral lung, heart and contralateral breast. There was no significant difference in hotspot volume between the two approaches. However, compared with the manual plan, the autoplan performed slightly better in ipsilateral-lungsparing (in terms of $\mathrm{V}_{16 \mathrm{~Gy}}, 14.0 \pm 3.6$ vs. $13.3 \pm 3.0, p<$ 0.01 , and mean dose, $6.6 \pm 1.5$ vs. $6.3 \pm 1.2, p<0.01$ ), but was inferior in contralateral-breast-sparing (in terms of maximal dose, $3.5 \pm 7.3$ vs.5.0 $\pm 8.7, p<0.01$ ). Additionally, the autoplan was generally superior to the manual plan in CI $(1.24 \pm 0.06$ vs. $1.30 \pm 0.09, p<0.01)$ and $\mathrm{MU}$ $(1010 \pm 46$ vs. $1205 \pm 187, p<0.01)$.

The average time to generate an autoplan was only five to 6 min irrespective of each patient's anatomical variance, while the manual planning time ranged from $1 \mathrm{~h}$ to one and a half hours. Figure 3 showed the autoplan and manual plan dose distributions for patients with different breast sizes (large, medium and small). Table 2 shows the ipsilateral lung, contralateral breast and heart 
Table 1 The mean, minimal and maximal value of the parameters for evaluation of PTV coverage and OARs constraints of all plans. Data analysis for comparison between manual plans and autoplans was done with paired two-tailed $t$-test. A $p$-value less than 0.05 was considered statistically significant

\begin{tabular}{|c|c|c|c|}
\hline & Manual & Auto & \\
\hline Parameter & Mean value \pm SD [Min, Max] & Mean value \pm SD [Min, Max] & $p$-value \\
\hline \multicolumn{4}{|l|}{ Body } \\
\hline$V_{110 \%}[\%]$ & $0.07 \pm 0.29[0.0,2.15]$ & $0.03 \pm 0.23[0.0,2.15]$ & 0.29 \\
\hline \multicolumn{4}{|l|}{ PTV } \\
\hline$V_{95 \%}[\%]$ & $98.5 \pm 0.5[96.7,99.5]$ & $98.5 \pm 0.6[96.4,99.9]$ & 0.40 \\
\hline \multicolumn{4}{|c|}{ Ipsilateral lung } \\
\hline$V_{16 G y}[\%]$ & $14.0 \pm 3.6[5.1,28.3]$ & $13.3 \pm 3.0[4.3,26.9]$ & $<0.01$ \\
\hline Mean[Gy] & $6.6 \pm 1.5[3.0,12.7]$ & $6.3 \pm 1.2[2.7,10.9]$ & $<0.01$ \\
\hline \multicolumn{4}{|l|}{ Heart } \\
\hline$V_{20 G y}[\%]$ & $0.90 \pm 1.30[0.0,8.0]$ & $0.90 \pm 1.10[0.0,4.4]$ & 0.79 \\
\hline Mean[Gy] & $1.4 \pm 0.6[0.5,4.0]$ & $1.4 \pm 0.6[0.6,3.3]$ & 0.30 \\
\hline \multicolumn{4}{|c|}{ Contralateral breast } \\
\hline$V_{5 G y}[\%]$ & $0.22 \pm 1.29[0.0,10.40]$ & $0.46 \pm 2.50[0.0,22.83]$ & 0.31 \\
\hline $\operatorname{Max}[G y]$ & $3.5 \pm 7.3[0.2,43.8]$ & $5.0 \pm 8.7[0.2,44.6]$ & $<0.01$ \\
\hline $\mathrm{Cl}$ & $1.30 \pm 0.09[1.10,1.63]$ & $1.24 \pm 0.06[1.14,1.41]$ & $<0.01$ \\
\hline $\mathrm{HI}$ & $1.08 \pm 0.02[1.05,1.13]$ & $1.07 \pm 0.02[1.05,1.13]$ & $<0.01$ \\
\hline $\mathrm{MU}$ & $1205 \pm 187[775,1650]$ & $1010 \pm 46[772,1353]$ & $<0.01$ \\
\hline
\end{tabular}

dose parameters for all plans stratified by the patients' breast sizes. In general, different breast sizes did not have much impact on the autoplan parameters, but for manual plans, there was slightly better ipsilateral-lungsparing in smaller breast $\left(\mathrm{V}_{4 \mathrm{~Gy}}=25.7 \pm 3.7 \%\right)$ than in larger $\left(\mathrm{V}_{4 \mathrm{~Gy}}=28.1 \pm 6.9 \%\right)$ or medium-sized $\left(\mathrm{V}_{4 \mathrm{~Gy}}=29.7 \pm\right.$ $5.8 \%)$ breast $(p<0.01)$.

For comparison purposes, the $\mathrm{V}_{95 \%}$ of the PTV for the manual plan was normalized to the $\mathrm{V}_{95 \%}$ of the PTV for the autoplan for each patient. As shown in Fig. 4, the DVH curves of autoplans and manual plans for the heart nearly overlapped. However, compared with the manual plans, the DVH curve of autoplans had a better ipsilateral-lung-sparing trend but less contralateralbreast-sparing.

\section{Discussion}

Manual treatment planning is an iteratively trial-anderror process that involves repeatedly adjusting beam angles and objective template parameters based on each treatment planner's experience, skill and knowledge. This manual approach results in high workload and potentially suboptimal plan quality due to the operatordependent preferences and priorities of the treatment planner and the physician. This study demonstrates that a well-designed automated treatment plan-generating algorithm can reduce treatment plan variability and standardize treatment plans. This approach results from more ideal, standardized gantry angle and collimator angle design with less inter-planner variability in autoplans. As it is never an easy task to convince medical personnel to embrace a new technology or automate clinical workflow, we designed this study to compare the manual plans and in-house-developed autoplans to examine the quality and feasibility of the automated plan-generating program for this large cohort study.

The auto-planning algorithm for whole-breast irradiation emulated the manual plan generation process, and required only five to $6 \mathrm{~min}$ to generate an optimized plan. Compared with the available published literature for automated breast irradiation planning, the autoplanning algorithm developed in this study saved time in terms of plan generation and optimization. A study published in 2010 used rapid but robust two-field tangential IMRT planning with a speed of 9 min per plan for 53 patients with the purpose of selecting patients that might benefit more from the deep inspiration breath hold technique [19]. A Netherland's study presented a single-click optimized autoplan software with planning time of $20 \mathrm{~min}$ for breast and prostate and $7 \mathrm{~min}$ for palliative vertebrae [14]. A Canadian study conducted by Purdie et al. used the Pinnacle treatment planning system to generate optimized whole-breast irradiation treatment plan using radio-opaque markers placed during CT simulation as the only input, with the mean time of 7 min per plan generation $[10,11]$.

Aside from the timesaving advantage of automated treatment planning, the above-mentioned studies 

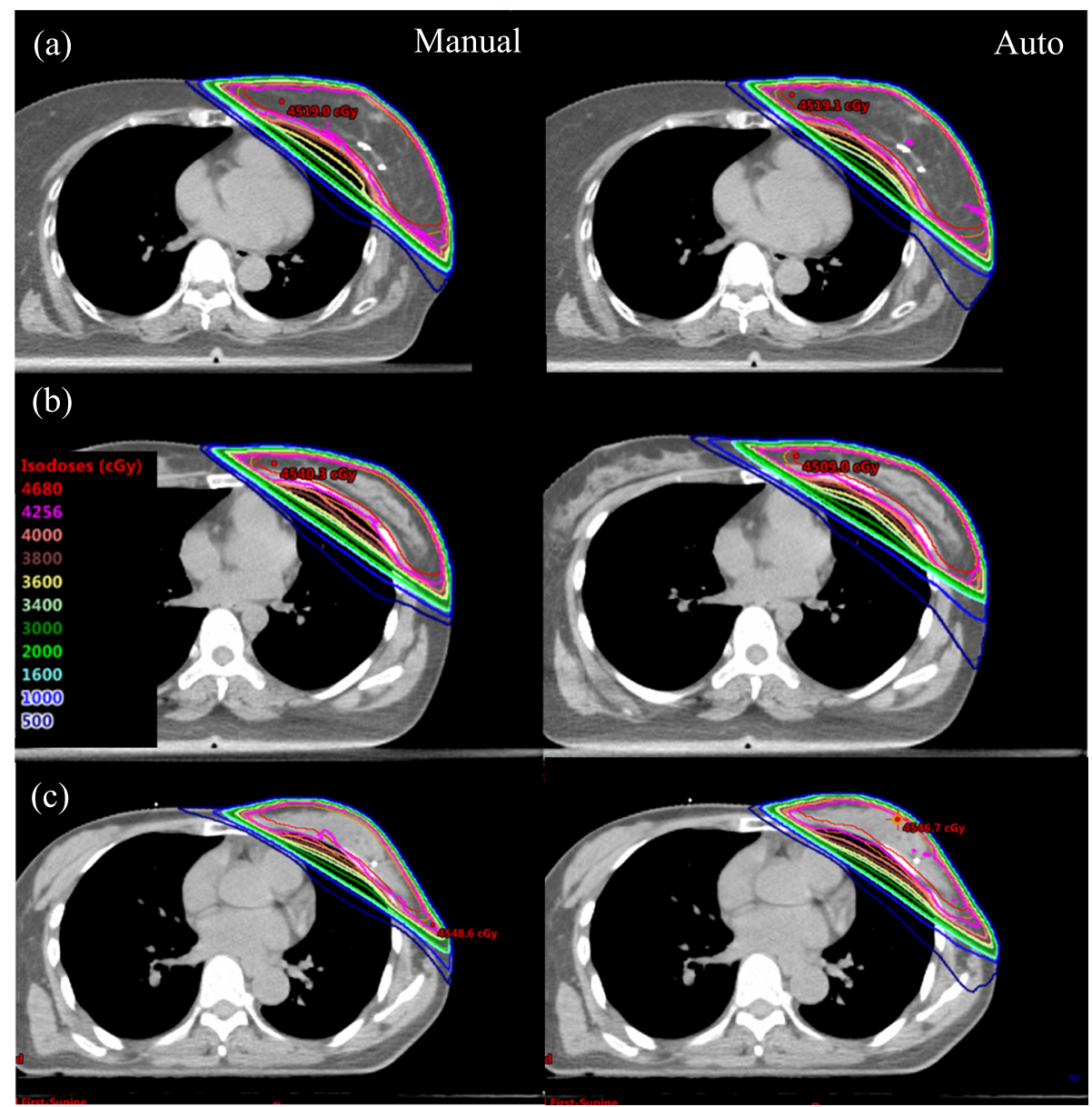

Fig. 3 Dose distributions in three representative patients with different breast sizes. Row (a) represents large breast size, row (b) medium breast size, and row (c) small breast size. Left column shows the dose distributions of autoplan, and right column the manual plan. All plans prescribed $42.56 \mathrm{~Gy}$ in 16 fractions. Isodose lines were drawn with different colors

generally showed equivalent autoplan clinical acceptability to manual plans with equivalent target dose and OAR constraint parameters, as well as similar treatment delivery time, or MUs. The advantage of applying the autoplans in this study include the following. First, the machine workload is minimized as the autoplans use fewer MUs to achieve similar PTV coverage and OAR sparing comparable with the manual plans overall. Other

Table 2 The constraints evaluation of ipsilateral lung, contralateral breast and heart of all plans stratified by different breast sizes. $L$ large breast size $(n=18), M$ medium breast size $(n=52)$, $S$ small breast size $(n=29)$

\begin{tabular}{|c|c|c|c|c|c|c|c|c|}
\hline \multirow[b]{2}{*}{ Parameter } & \multicolumn{4}{|c|}{ Manual (Mean value \pm SD) } & \multicolumn{4}{|c|}{ Auto (Mean value \pm SD) } \\
\hline & $\mathrm{L}$ & M & $\mathrm{S}$ & $\overline{p \text {-value }}$ & L & M & S & $p$-value \\
\hline \multicolumn{9}{|l|}{ Ipsilateral lung } \\
\hline $\mathrm{V}_{16 \mathrm{~Gy}}[\%]$ & $12.9 \pm 3.8$ & $14.5 \pm 3.6$ & $13.7 \pm 3.5$ & 0.25 & $13.0 \pm 3.5$ & $13.5 \pm 3.3$ & $13.1 \pm 2.2$ & 0.78 \\
\hline $\mathrm{V}_{4 \mathrm{~Gy}}[\%]$ & $28.1 \pm 6.9$ & $29.7 \pm 5.8$ & $25.7 \pm 3.7$ & $<0.01$ & $28.8 \pm 7.0$ & $28.4 \pm 5.2$ & $26.8 \pm 2.4$ & 0.30 \\
\hline \multicolumn{9}{|c|}{ Contralateral breast } \\
\hline $\mathrm{V}_{5 \mathrm{~Gy}}[\%]$ & $0.0 \pm 0.0$ & $0.3 \pm 1.7$ & $0.0 \pm 0.4$ & 0.46 & $0.0 \pm 0.0$ & $0.8 \pm 3.4$ & $0.0 \pm 0.0$ & 0.23 \\
\hline $\operatorname{Max}[\mathrm{Gy}]$ & $2.0 \pm 2.3$ & $4.7 \pm 9.6$ & $2.2 \pm 2.6$ & 0.19 & $3.6 \pm 4.4$ & $6.6 \pm 11.3$ & $2.8 \pm 2.9$ & 0.13 \\
\hline \multicolumn{9}{|l|}{ Heart } \\
\hline$V_{20 G y}[\%]$ & $0.9 \pm 1.4$ & $0.9 \pm 1.3$ & $0.9 \pm 1.2$ & 0.98 & $1.2 \pm 1.5$ & $0.9 \pm 0.9$ & $0.9 \pm 1.1$ & 0.42 \\
\hline Mean[Gy] & $1.5 \pm 0.6$ & $1.4 \pm 0.6$ & $1.3 \pm 0.7$ & 0.37 & $1.7 \pm 0.7$ & $1.4 \pm 0.5$ & $1.2 \pm 0.5$ & 0.03 \\
\hline
\end{tabular}



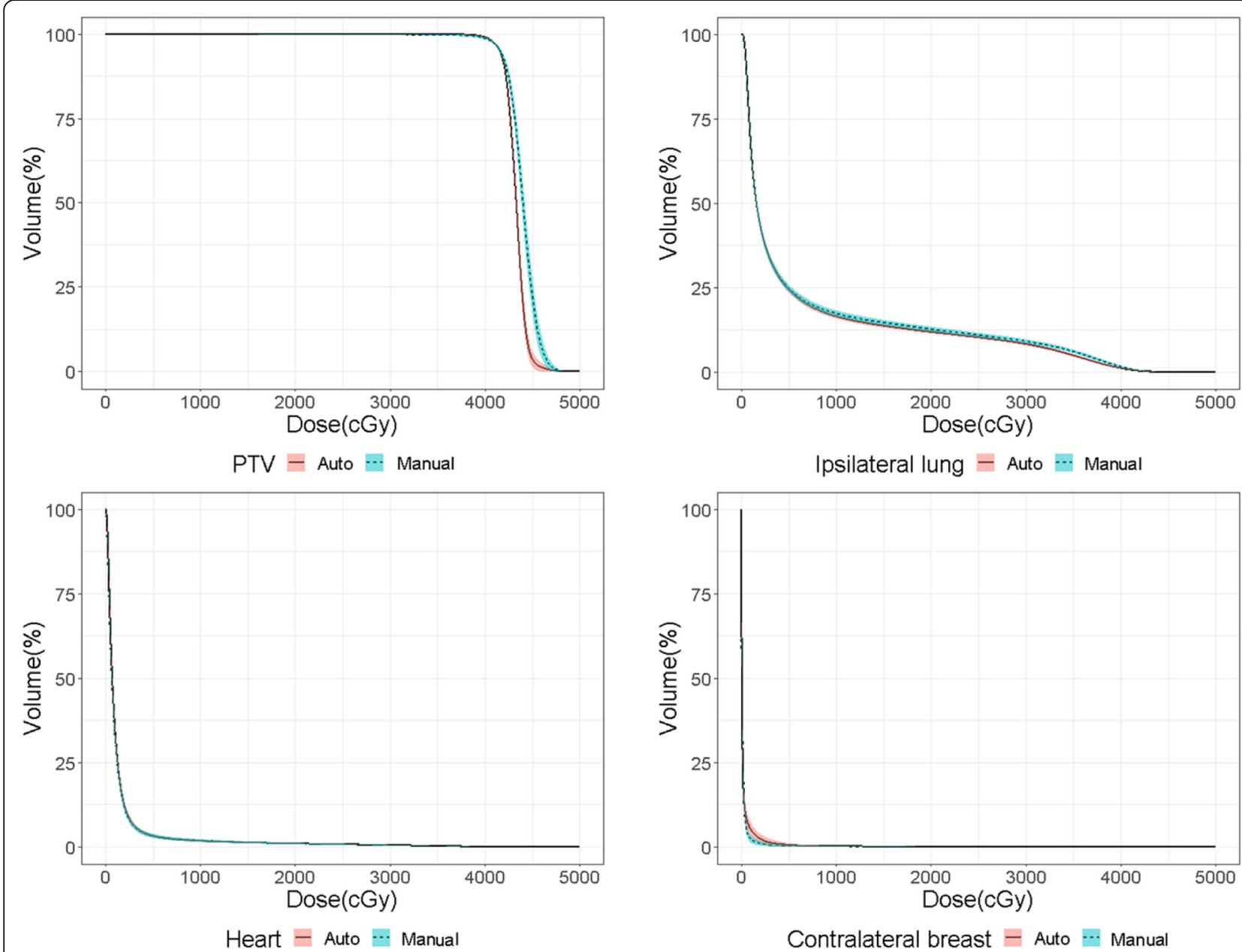

Fig. 4 Mean DVH curves with 95\% confidence interval (shaded area) for the PTV, ipsilateral lung, heart and contralateral breast for autoplans (solid red lines) and manual plans (dashed green lines)

previously mentioned published studies used two-field or four-field design, with no difference in MUs between automated plans and manual plans. Second, there is a slightly higher homogeneity in PTV dose coverage for autoplans. The DVH analyses (Fig. 4) demonstrate a steeper slope in the average DVH curve for the PTV of the autoplans compared with the manual plans. Third, the OAR dose parameters and target coverage are uniform and consistent in autoplans than in manual plans. The narrow 95\% confidence interval shown in the DVH analyses of the 99 autoplans indicates the uniform consistency of the dose parameters.

The automated planning algorithm in this study is highly efficient as it uses six-field IMRT and could generate an optimized plan with high conformity and homogeneity in five to $6 \mathrm{~min}$ on a standalone workstation. The observed advantage of autoplans implies that when applying a more complicated field design, an automated planning algorithm may not only save much time for medical physicists and dosimetrists, but also find acceptable or even better solutions for a standardized and optimized treatment plan than a human could within a limited time. Four to five hypofractionated breast cancer treatments are planned per week at our oncology department on average. The estimated working hours saved per month is therefore $18 \mathrm{~h}$ per month. Moreover, a multi-field complex planning task requires great experience, many calculations and much trial-and-error, which may be overwhelming in difficult cases. Our automated planning algorithm achieves the best gantry angles, collimator angles, MUs for each field and minimal hotspots through iteration and optimization. This would be a daunting task if reproduced manually. Applying an automated planning algorithm can also reduce interplanner variability between experienced and nonexperienced treatment planners, which may contribute to plan quality control and educational training.

It is also interesting to note that, as shown in the DVH curves in Fig. 4 and parameters in Table 1, the 
autoplanning algorithm tends to maximize the PTV coverage without compromising it in order to spare the lung dose, which probably results from the strict adherence to the planning algorithm design (Fig. 1) and the objective template (Additional file 1: Table S1). In reality, human doctors and physicists might accept a tradeoff between the PTV coverage, lung dose and other OAR constraints, considering a patient's medical or clinical condition, such as chronic lung disease or impaired heart function. As the criterion for plan approval is according to each clinician's discretion, our autoplanning algorithm has the advantage of quickly generating several optimized plans using different objective templates, allowing clinicians to choose the ideal one to approve for treatment delivery based on clinical judgement. However, physicist manual refinement of automatically generated plan may be required based on individual patient's condition.

To date, automated workflow has been developed and implemented in various businesses but the pace of applying automation into health care is not as fast as expected. In the recent two decades, the rapid progress in computerized radiotherapy treatment planning systems has led to great interest in the possibility of automating radiotherapy workflow, which includes automated target delineation (auto-segmentation), automated treatment planning, automated real-time adaptive radiotherapy and automated quality assurance [20-25]. This study presents a well-designed fully automated planning algorithm for standardized whole-breast radiotherapy treatment plan generation with a large cohort of 99 patients. By retrospectively analyzing and comparing the autoplans and manual plans, we prove that this algorithm has great potential in clinical applicability, and may improve radiotherapy treatment planning workflow in efficiency and standardization. While the autoplanning algorithm may save much time and reduce the repetitive planning workload of medical physicists and dosimetrists if implemented clinically, it may also help medical personnel focus on tasks requiring innovation and creativity. In the future, we seek to develop a program that automatically extracts clinical information and combine it with the automated planning algorithm to develop a personalized automated treatment planning program, thereby increasing its applicability.

\section{Conclusions}

An automated treatment-planning algorithm for standardized and optimized hypofractionated whole-breast irradiation plan generation is developed to reduce the radiation oncology personnel workload and improve human performance and productivity. Autoplans with higher efficacy (more MU-saving) and with more uniformity and consistency in plan parameters are generated by the algorithm when compared with manual plans. We conclude that a well-designed automated treatment planning program can improve current clinical practice in the radiation oncology field, and argue that automatic treatment planning will become part of the standard workflow in radiation oncology departments at medical centers.

\section{Supplementary information}

Supplementary information accompanies this paper at https://doi.org/10. 1186/s13014-020-1468-9.

Additional file 1 : Table $\mathbf{S 1}$. The objective template for dosimetry optimization.

\section{Abbreviations}

Cl: Conformity index; CT: Computerized tomography; CTV: Clinical target volume; DIBH: Deep-inspiration breath-hold; DVH: Dose-volume histogram; ESAPI: Eclipse scripting application programming interface; HI: Homogeneity index; IMRT: Intensity-modulated radiation therapy; MU: Monitor unit; OAR: Organ at risk; PTV: Planning target volume; QUANTEC: Quantitative analysis of normal tissue effects in the clinic; RTOG: Radiation therapy oncology group; SD: Standard deviation; TPS: Treatment planning system

\section{Acknowledgements}

Not applicable.

\section{Authors' contributions}

$C Y L, K C L$ and $T H W$ designed the autoplanning algorithm. CYL and TCL collected the data, performed the statistical analysis and wrote the manuscript. ACS, THW, JHJ, LCL and JAL revised and approved the final manuscript. All authors read and approved the final manuscript.

\section{Funding}

This research is partially supported by CMUH Grant DMR-108-055.

\section{Availability of data and materials}

The autoplanning code during the current study are available from CY Lin (cylin1230@gmail.com) to any reader directly on reasonable request.

\section{Ethics approval and consent to participate}

Data collection and analysis were approved by the institutional review board of China Medical University Hospital, Taichung, Taiwan (CMUH106-REC3-119).

Consent for publication

Not applicable.

Competing interests

The authors declare that they have no competing interests.

\section{Author details}

'Department of Radiation Oncology, China Medical University Hospital, China Medical University, Taichung, Taiwan. ${ }^{2}$ Department of Medicine, China Medical University, Taichung, Taiwan. ${ }^{3}$ Department of Biomedical Imaging and Radiological Sciences, National Yang-Ming University, Taipei, Taiwan. ${ }^{4}$ Department of Biomedical Imaging and Radiological Science, China Medical University, Taichung, Taiwan. ${ }^{5}$ Department of Surgery, China Medical University Hospital, Taichung, Taiwan.

Received: 18 November 2019 Accepted: 15 January 2020 Published online: 17 March 2020

\section{References}

1. Guo F, Kuo Y, Berenson AB. Breast Cancer incidence by stage before and after change in screening guidelines. Am J Prev Med. 2019;56:100-8.

2. Lin $R$, Tripuraneni P. Radiation therapy in early-stage invasive breast Cancer. Indian J Surg Oncol. 2011;2:101-11. 
3. Yarnold J, Bentzen SM, Coles C, Haviland J. Hypofractionated whole-breast radiotherapy for women with early breast Cancer: myths and realities. Int J Radiat Oncol. 2011;79:1-9.

4. Quantitative Analyses of Normal Tissue Effects in the Clinic (QUANTEC): An Introduction to the Scientific Issues - International Journal of Radiation Oncology • Biology • Physics. Available at: https://www.redjournal.org/ article/S0360-3016(09)03300-8/fulltext. Accessed 11 May 2019.

5. Rudra S, Al-Hallaq HA, Feng C, Chmura SJ, Hasan Y. Effect of RTOG breast/ chest wall guidelines on dose-volume histogram parameters. J Appl Clin Med Phys Am Coll Med Phys. 2014;15:4547.

6. Kim H, et al. Automated field-in-field (FIF) plan framework combining scripting application programming Interface and user-executed program for breast forward IMRT. Technol Cancer Res Treat. 2018;17:153303381881039.

7. Jain $\mathrm{P}$, et al. Inter-fraction motion and dosimetric consequences during breast intensity-modulated radiotherapy (IMRT). Radiother Oncol. 2009:90:93-8.

8. Kestin $\mathrm{LL}$, et al. Intensity modulation to improve dose uniformity with tangential breast radiotherapy: initial clinical experience. Int J Radiat Oncol. 2000;48:1559-68

9. Ayata HB, Güden M, Ceylan C, Kücük N, Engin K. Comparison of dose distributions and organs at risk (OAR) doses in conventional tangential technique (CTT) and IMRT plans with different numbers of beam in leftsided breast cancer. Rep Pract Oncol Radiother. 2011;16:95-102.

10. Purdie TG, Dinniwell RE, Letourneau D, Hill C, Sharpe MB. Automated planning of tangential breast intensity-modulated radiotherapy using heuristic optimization. Int J Radiat Oncol. 2011;81:575-83.

11. Purdie TG, Dinniwell RE, Fyles A, Sharpe MB. Automation and intensity modulated radiation therapy for individualized high-quality tangent breast treatment plans. Int J Radiat Oncol. 2014;90:688-95.

12. Hansen $\mathrm{CR}$, et al. Automatic treatment planning improves the clinical quality of head and neck cancer treatment plans. Clin Transl Radiat Oncol. 2016;1:2-8,

13. Hazell I, et al. Automatic planning of head and neck treatment plans. J Appl Clin Med Phys. 2016;17:272-82.

14. De Graaf $R$, et al. EP-1628: single-click automatic radiotherapy treatment planning for breast, prostate and vertebrae. Radiother Oncol. 2016;119:5758-9.

15. Buergy $\mathrm{D}$, et al. Fully automated treatment planning of spinal metastases a comparison to manual planning of volumetric modulated arc therapy for conventionally fractionated irradiation. Radiat Oncol. 2017;12:33.

16. Higher Per Daily Treatment-Dose Radiation Therapy or Standard Per Daily Treatment Radiation Therapy in Treating Patients With Early-Stage Breast Cancer That Was Removed by Surgery - Tabular View ClinicalTrials.gov. Available at: https://clinicaltrials.gov/ct2/show/record/ NCT01349322. Accessed 7 May 2019.

17. Kataria T, Sharma K, Subramani V, Karrthick KP, Bisht SS. Homogeneity index: an objective tool for assessment of conformal radiation treatments. J Med Phys Assoc Med Phys. 2012;37:207-13.

18. Feuvret L, Noël G, Mazeron J-J, Bey P. Conformity index: a review. Int J Radiat Oncol Biol Phys. 2006;64:333-42.

19. Wang W, et al. Rapid automated treatment planning process to select breast Cancer patients for active breathing control to achieve cardiac dose reduction. Int J Radiat Oncol. 2012;82:386-93.

20. Men $\mathrm{K}$, et al. Fully automatic and robust segmentation of the clinical target volume for radiotherapy of breast cancer using big data and deep learning. Phys Med. 2018;50:13-9.

21. Liu C, et al. An automated dose tracking system for adaptive radiation therapy. Comput Methods Prog Biomed. 2018;154:1-8.

22. Lamb J, et al. Online Adaptive Radiation Therapy: Implementation of a New Process of Care. Cureus. 9(8):e1618.

23. Krayenbuehl J, Di Martino M, Guckenberger M, Andratschke N. Improved plan quality with automated radiotherapy planning for whole brain with hippocampus sparing: a comparison to the RTOG 0933 trial. Radiat Oncol. 2017;12:161

24. Cai B, et al. Standardization and automation of quality assurance for highdose-rate brachytherapy planning with application programming interface. Brachytherapy. 2019;18:108-114.e1.

25. Sarkar B, et al. Standardization of volumetric modulated arc therapy-based frameless stereotactic technique using a multidimensional ensemble-aided knowledge-based planning. Med Phys. 2019;46:1953-62.

\section{Publisher's Note}

Springer Nature remains neutral with regard to jurisdictional claims in published maps and institutional affiliations.

\section{Ready to submit your research? Choose BMC and benefit from}

- fast, convenient online submission

- thorough peer review by experienced researchers in your field

- rapid publication on acceptance

- support for research data, including large and complex data types

- gold Open Access which fosters wider collaboration and increased citations

- maximum visibility for your research: over $100 \mathrm{M}$ website views per year

At BMC, research is always in progress.

Learn more biomedcentral.com/submissions 